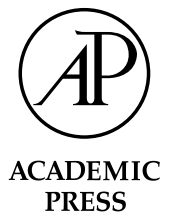

\title{
Cloning and sequencing of wsp encoding gene fragments reveals a diversity of co-infecting Wolbachia strains in Acromyrmex leafcutter ants
}

\author{
S. Van Borm, ${ }^{a, *}$ T. Wenseleers, ${ }^{\text {a,b }}$ J. Billen, ${ }^{\text {a }}$ and J.J. Boomsma ${ }^{c}$ \\ ${ }^{a}$ Zoological Institute, Laboratory of Entomology, Katholieke Universiteit Leuven, Naamsestraat 59, B-3000 Leuven, Belgium \\ ${ }^{\mathrm{b}}$ Department of Animal and Plant Sciences, Sheffield University, Western Bank, Sheffield S10 2TN, UK \\ ${ }^{\mathrm{c}}$ Zoological Institute, University of Copenhagen, Universitetsparken 15, 2100 Copenhagen, Denmark
}

Received 20 February 2002; received in revised form 27 June 2002

\begin{abstract}
By sequencing part of the wsp gene of a series of clones, we detected an unusually high diversity of nine Wolbachia strains in queens of three species of leafcutter ants. Up to four strains co-occurred in a single ant. Most strains occurred in two clusters (InvA and InvB), but the social parasite Acromyrmex insinuator hosted two additional infections. The multiple Wolbachia strains may influence the expression of reproductive conflicts in leafcutter ants, but the expected turnover of infections may make the cumulative effects on host ant reproduction complex. The additional Wolbachia infections of the social parasite A. insinuator were almost certainly acquired by horizontal transmission, but may have facilitated reproductive isolation from its closely related host.
\end{abstract}

(c) 2002 Elsevier Science (USA). All rights reserved.

\section{Introduction}

Wolbachia pipientis is a Rickettsia-like $\alpha$-proteobacterium that infects a wide range of arthropods (O'Neill et al., 1997; Werren, 1997). It is known to selfishly enhance its matrilineal transmission through various alterations of host reproduction, including feminisation (reviewed by Rigaud, 1997), thelytokous parthenogenesis (reviewed by Stouthamer, 1997), male-killing (MK, reviewed by Stevens et al., 2001) and cytoplasmic incompatibility (CI, reviewed in Hoffmann and Turelli, 1997). Recently, fecundity enhancing effects have also been reported (Dedeine et al., 2001). Wolbachia therefore covers the entire range of endosymbiotic interactions, from parasitism to mutualism. Recent phylogenetic studies, based on $16 \mathrm{~S}$ rDNA and faster evolving protein-encoding genes ( $w s p$, ftsZ, and GroE) indicate that Wolbachia contains at least four major clades. Two are found in arthropods and are designated A and B (Werren et al., 1995b; Zhou et al., 1998). The other divisions (C and D) are found in nem-

\footnotetext{
${ }^{*}$ Corresponding author. Fax: +32-16-324575.

E-mail address: Steven.Vanborm@bio.kuleuven.ac.be (S. Van Borm).
}

atodes (Bandi et al., 1998). A $16 \mathrm{~S}$ rDNA sequence in a collembolan suggests a possible fifth group E (Vandekerckhove et al., 1999). In insects, Wolbachia is a widespread and common endosymbiont. PCR-based screenings have shown that $16 \%$ of a large sample of neotropical insects are Wolbachia infected (Werren et al., 1995a), and similar figures have been found in a sample of palearctic (West et al., 1998) and nearctic insects (Werren and Windsor, 2000). Using an ultra-sensitive assay, figures up to $76 \%$ have been obtained for nearctic arthropods (Jeyaprakash and Hoy, 2000).

Previous work on ants has shown that the majority of species screened so far carry Wolbachia infections: 25 out of $50(50 \%)$ Indo-Australian ants (Wenseleers et al., 1998), 10 out of $10(100 \%)$ Nearctic ants (Jeyaprakash and Hoy, 2000), and 7 out of $7(100 \%)$ Panamanian leafcutter ants (Van Borm et al., 2001) have now been shown to harbour Wolbachia. The high incidence of Wolbachia in ants might be due to the more favourable conditions for invasion and maintenance in haplodiploid social hosts compared to solitary hosts (Wenseleers et al., 1998). For leafcutter and wood ants, Wolbachia has also been shown to be present at or near to fixation (Van Borm et al., 2001; Wenseleers et al., 2001). Specific PCR has 
demonstrated that both the A and B subgroup Wolbachia occur in ants (Jeyaprakash and Hoy, 2000; Shoemaker et al., 2000; Van Borm et al., 2001), but until now it has remained unclear how many distinct strains they represent. Sex-specific infection patterns and theoretical models are consistent with the Wolbachia strains in three species of Panamanian Acromyrmex leafcutter ants being either of the cytoplasmatic incompatibility (CI) type or of the "Male-killing" (MK) type (Van Borm et al., 2001). Detailed genetic characterisation of the strains involved may strengthen this conclusion if they cluster with strains that have these effects. These inferences are important because ants, in contrast to many other arthropods, can normally not be reared across generations in the laboratory, so that experimental crosses of infected and uninfected lineages are not available as tools to demonstrate the phenotypic effect of Wolbachia infections.

In the present study, we determine the number and the phylogenetic identity of Wolbachia strains present in the three sympatric Panamanian leafcutter ant species, $A c$ romyrmex octospinosus, Acromyrmex echinatior and Acromyrmex insinuator. A. octospinosus is a common and widespread ant species in Panama, while A. echinatior and $A$. insinuator are rarer and more patchily distributed (J.J.B., unpubl. data). A. insinuator is a social parasite and can only successfully reproduce within $A$. echinatior colonies and not in colonies of the sympatric $A$. octospinosus (Bekkevold and Boomsma, 2000; Schultz et al., 1998). To characterise the Wolbachia strains that are present, we amplified, cloned and sequenced the gene that encodes the Wolbachia cell surface protein wsp. In a previous study, using strain specific primers, it was shown that both A and B strains were present (Van Borm et al., 2001). Using the present approach we can now assess the full diversity of strains that are present and accurately place them within the Wolbachia phylogeny.

\section{Methods}

\subsection{Sampling and DNA extraction}

Gynes (winged prospective queens) of A. octospinosus, A. echinatior and A. insinuator were collected in Gamboa, Panama, and preserved in $95 \%$ ethanol in April 2000. To minimise the risk of cross-contamination, the pre-extraction treatment and all DNA extraction procedures were performed under sterile conditions in a laminar flow hood. After taking them out of their collection tubes, five gynes (prospective queens; each from a different colony) of each species were externally sterilised by immersion in 70\% ethanol, followed by two rinses in double distilled water and exposure to $250 \mathrm{~nm}$ UV radiation for $15 \mathrm{~min}$. The entire ants were subsequently ground after freezing in liquid nitrogen, after which their DNA was extracted by $3 \mathrm{~h}$ incubation at $55^{\circ} \mathrm{C}$ and $20 \mathrm{~min}$ boiling in $500 \mu \mathrm{l}$ of a $10 \%$ Biorad Chelex 100 resin solution. The samples were centrifuged and stored at $-20^{\circ} \mathrm{C}$ until use.

\subsection{General PCR amplification}

General primers $w s p 81 \mathrm{~F} 5^{\prime} \mathrm{TGG}$ TCC AAT AAG TGA TGA AGA AAC $3^{\prime}$ and wsp691R $5^{\prime}$ AAA AAT TAA ACG CTA CTC CA 3' (Braig et al., 1998) were used to amplify a 586-617 bp stretch of the gene encoding the $W$. pipientis surface protein $w s p$. PCR amplification was carried out according to the protocol of Pintureau et al. (2000). After amplification, $10 \mu 1$ of the reaction mixture was electrophoresed with a $100 \mathrm{bp}$ DNA ladder size standard (GibcoBRL) on 1.5\% agarose minigels. DNA bands were visualized by ethidium bromide staining. A specimen of the ant Gnamptogenys menadensis (Sulawesi) known to contain an A strain Wolbachia (Wenseleers and Billen, 2000) was included as a positive control in every amplification. For each species, we selected one Wolbachia positive gyne for cloning and sequencing of the PCR product.

\subsection{Cloning}

PCR amplification products were ligated without further purification into a pCR 2.1-TOPO vector (Invitrogen Topo TA Cloning Kit). Subsequently the vectors were transformed in chemically competent Escherichia coli cells (Invitrogen Topo TA Cloning Kit), plated on selective agar plates containing ampicillin and incubated overnight at $37^{\circ} \mathrm{C}$. The resulting clones were suspended in $50 \mu \mathrm{l}$ double distilled water. Positive transformants were determined by PCR, using primers M13F ( $5^{\prime}$ GTA AAA CGA CGG CCA G 3') and M13R (5' CAG GAA ACA GCT ATG AC $3^{\prime}$ ) provided by the manufacturer. PCR amplification reactions were carried out in $25 \mu \mathrm{l}$ reaction mixtures, which contained $0.8 \mu \mathrm{M}$ of each primer, $0.2 \mathrm{mM}$ of each dNTP, $1.5 \mathrm{mM} \mathrm{MgCl}_{2}, 1 \mu$ of the suspended clone, $0.3 \mathrm{U}$ of Taq DNA polymerase (AmpliTaq, Perkin-Elmer Cetus) and PCR buffer specified by the manufacturer. PCR was performed with an initial denaturation at $97^{\circ} \mathrm{C}$ for $5 \mathrm{~min}$, followed by 30 cycles consisting of $95^{\circ} \mathrm{C}$ for $30 \mathrm{~s}, 60^{\circ} \mathrm{C}$ for $45 \mathrm{~s}$, and $72{ }^{\circ} \mathrm{C}$ for $1 \mathrm{~min}$, and a final $10 \mathrm{~min}$ extension at $72^{\circ} \mathrm{C}$.

\subsection{RFLP}

To distinguish distinct clones, restriction fragment length polymorphisms were determined by incubating $10 \mu \mathrm{l}$ of the M13-PCR product, $0.5 \mu \mathrm{l}$ of $10 \times$ buffer provided by the manufacturer and $1 \mu$ of restriction enzyme EcoRI or HindIII (MBI Fermentas) at $37^{\circ} \mathrm{C}$ for $4 \mathrm{~h}$. Of each resulting RFLP type (a group of identical clones representing a single Wolbachia strain), one was sequenced from the purified M13-PCR product. 


\subsection{Sequencing}

DNA was purified from the M13-PCR product (GFX PCR DNA and Gel Band Purification Kit, Amersham Pharmacia Biotech). Sequencing reactions contained 4 pmol of each IRD (infrared-dye)-labeled M13 primer, $5 \mathrm{U}$ of SequiTherm ExcelII DNA polymerase (Epicentre Technologies) and buffer prescribed by the manufacturer. The PCR reaction was performed with an initial denaturation at $95^{\circ} \mathrm{C}$ for $5 \mathrm{~min}$, followed by 30 cycles of $95^{\circ} \mathrm{C}$ for $30 \mathrm{~s}, 55^{\circ} \mathrm{C}$ for $15 \mathrm{~s}$ and $70{ }^{\circ} \mathrm{C}$ for $1 \mathrm{~min}$, and a final $10 \mathrm{~min}$ extension at $70^{\circ} \mathrm{C}$. Subsequently, reactions were transferred to a LI-COR automated sequencer for PAGE.

\subsection{Phylogenetic analysis}

The partial wsp gene sequences were aligned using the CLUSTAL W program (Thompson et al., 1994) followed by manual refinements taking translation into account. A $50 \mathrm{bp}$ region (positions 533-583) corresponding to the third hyper-variable region of the gene (Braig et al., 1998) could not be aligned with certainty and was deleted from the analysis. The resulting data set included 555 nucleotide sites (of which 177 were variable). Sequences were subsequently aligned to a subset of previously published Wolbachia sequences from other insects and arthropods (24 sequences found in GenBank). A distance matrix was calculated with $M E G A$ version 2.0, using a Kimura two-parameter distance metric and pairwise deletion of insertions and deletions (Kumar et al., 2001). From this matrix, a neighbourjoining tree was produced. Bootstrap analysis, using 1000 pseudoreplicates, was used to test the reliability of the clades in the phylogeny. In addition, we performed a maximum parsimony analysis, also using $M E G A$ version 2.0 (Kumar et al., 2001), bootstrapped with 500 pseudoreplicates. Finally, PHYLIP 3.57c (Felsenstein, 1995) was used for a maximum likelihood analysis, using a bootstrap analysis with 500 pseudoreplicates. The trees were rooted using the A Wolbachia clade as the sister group of the B Wolbachia clade. The phylogenetic organisation of insect-associated Wolbachia in two subgroups, A and B, has been confirmed in fts $Z$ (Werren et al., 1995b), wsp (Zhou et al., 1998) and GroE (Masui et al., 1997) sequencing studies.

\section{Results}

\subsection{Cloning and sequencing}

We picked up nine clones with an insert of the expected size in a single gyne of $A$. insinuator, 25 clones in a gyne of $A$. octospinosus, and 13 clones in an $A$. echinatior gyne, adding up to a total of 47 positive trans- formants. Among these clones, we found three distinct RFLP types for A. insinuator, four types for A. octospinosus and three for A. echinatior. We interpreted each RFLP type as a group of identical clones representing a single $W$. pipientis strain, and determined the partial wsp sequence for one clone belonging to each type. The sequences of two A. echinatior clones were almost identical (sequence divergence $0.29 \%$ ). We assumed that these differences were likely due to polymerase errors, and used only the consensus sequence of these two (' $A$. echinatior Bcons' in Fig. 1 and Table 1). The sequences of the remaining eight RFLP types were considerably more different (sequence divergence $>1 \%$, Table 1 ) or resided in different host species, resulting in a total of nine recognised and distinct Wolbachia strains: three in A. insinuator, four in A. octospinosus, and two in $A$. echinatior. Their wsp sequences were submitted to the GenBank database and are available under the accession numbers given in Fig. 1.

The results of the neighbour-joining analysis are shown in Fig. 1. The trees based on maximum likelihood and parsimony analyses are not shown, but do not significantly deviate from these results, apart from some minor changes in the interior branching pattern within clusters $I n v A$ and $I n v B$. Each of the two free-living (i.e. non-parasitic) species (A. octospinosus and A. echinatior) has one A Wolbachia strain and 1-3 B Wolbachia strains. The A Wolbachia of A. echinatior and A. octospinosus cluster together with other ant Wolbachia (Solenopsis richteri and Solenopsis invicta) in a wellsupported monophyletic group. We propose to name this subgroup InvA, after the A Wolbachia of S. invicta which was the first sequence described in this group (Shoemaker et al., 2000). The social parasite (A. insinuator) Wolbachia A is different from this cluster and groups together with Wolbachi a from Muscidifurax parasitoid wasps (subgroup InsA). A similar infectionpattern was found for the Wolbachia B strains. One well-supported group of ant Wolbachia (for which we propose the designation $\operatorname{InvB}$ ) consists of three A. octospinosus Wolbachia, one A. echinatior Wolbachia, one $A$. insinuator Wolbachia, and one $S$. invicta strain (which was the first to be described, Shoemaker et al., 2000). A second Wolbachia B strain of the social parasite $A$. insinuator is phylogenetically isolated from the $\operatorname{Inv} B$ group and clusters together with a Wolbachia strain of the parasitoid wasp T. nawai (Naw subgroup, Arakaki et al., 2000). The Bootstrap support values of the parsimony analysis confirm the robustness of most Acromyrmex Wolbachia clusters, with values of 99, 97, and 99 for the clusters InvB, Naw, and InvA, respectively. Confirmation of the InsA cluster (59) was less good, but it remained unambiguous that this clade is differentiated from the InvA clade. Differences between several strains in the $\operatorname{InvB}$ cluster are rather small, but always well above $1.1 \%$ within the same host ant, i.e. above the 


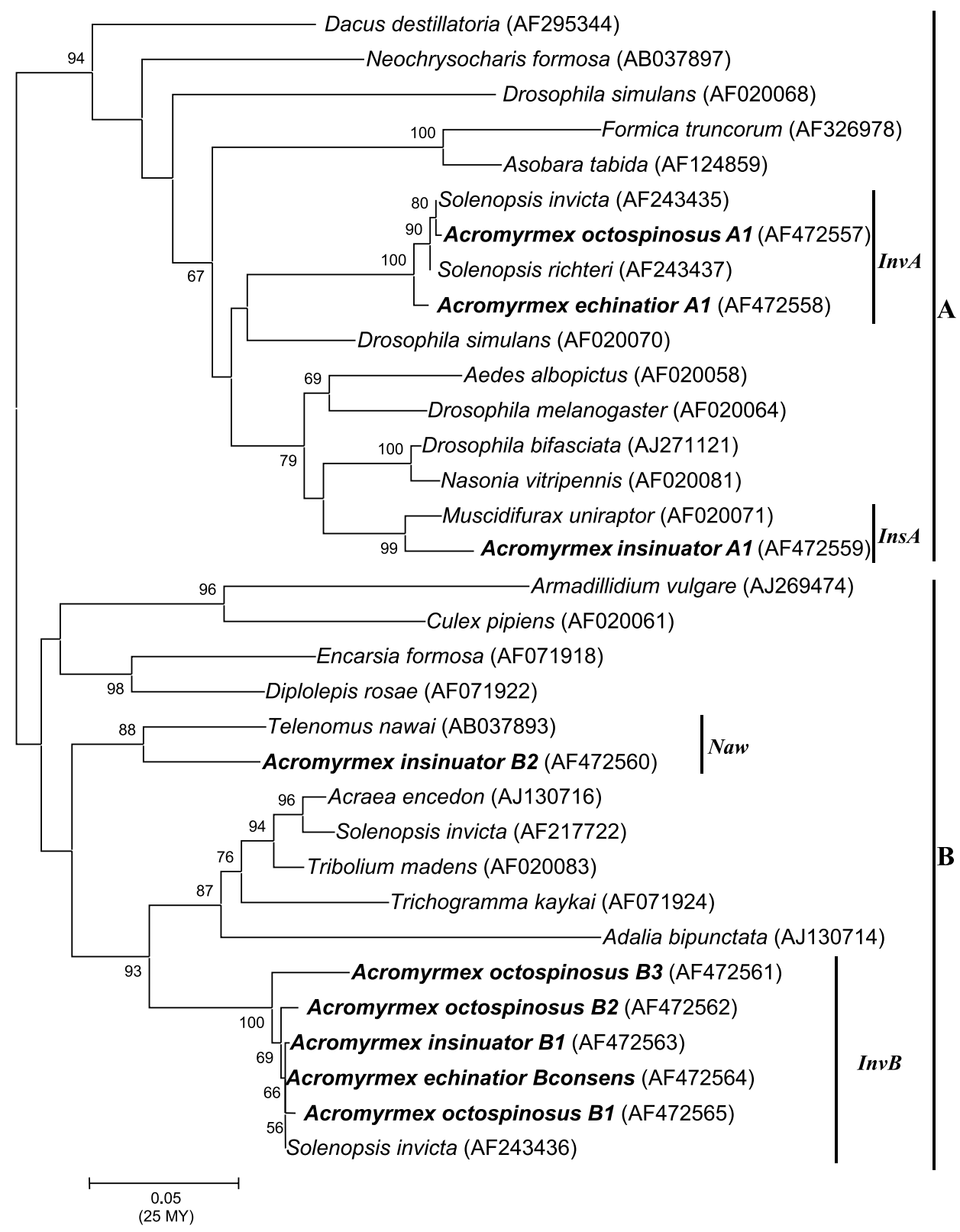

Fig. 1. Neighbour-joining tree for Wolbachia strains based on partial sequences of the wsp gene. Distances were calculated using the Kimura twoparameter model in MEGA 2.0. Bootstrap support values (1000 replicates) over 50\% are shown above the branches. Wolbachia strains are identified by the host species from which they were isolated, followed by a GenBank accession number. Sequences generated in this study are indicated in bold. The bar represents a distance of 0.050 (about $25 \mathrm{MY}$ if a $w s p$ evolutionary rate of $0.2 \%$ per MY is assumed, Wenseleers et al., 2001).

expected cloning error rate for a $500-600$ bp fragment. They therefore represent genuine strains and do not merely reflect sequencing errors.

\section{Discussion}

Previous work has documented the widespread occurrence of Wolbachia in ant species (Jeyaprakash and Hoy, 2000; Wenseleers et al., 1998) and has demonstrated a high prevalence of these symbionts in colonies of Atta and Acromyrmex leafcutter ants and Formica wood ants (Van Borm et al., 2001; Wenseleers et al., 2001). In the present study we demonstrate a highly complex diversity of strains occurring in single individuals of three Acromyrmex leafcutter ant hosts. Up to four different Wolbachia strains were detected in a single female (gyne), which to our knowledge is the highest number of co-infecting strains reported in a single insect so far. Multiple Wolbachia infections have been widely reported, but have as yet been restricted to either double (e.g., Breeuwer et al., 1992; Malloch et al., 2000; Rousset 


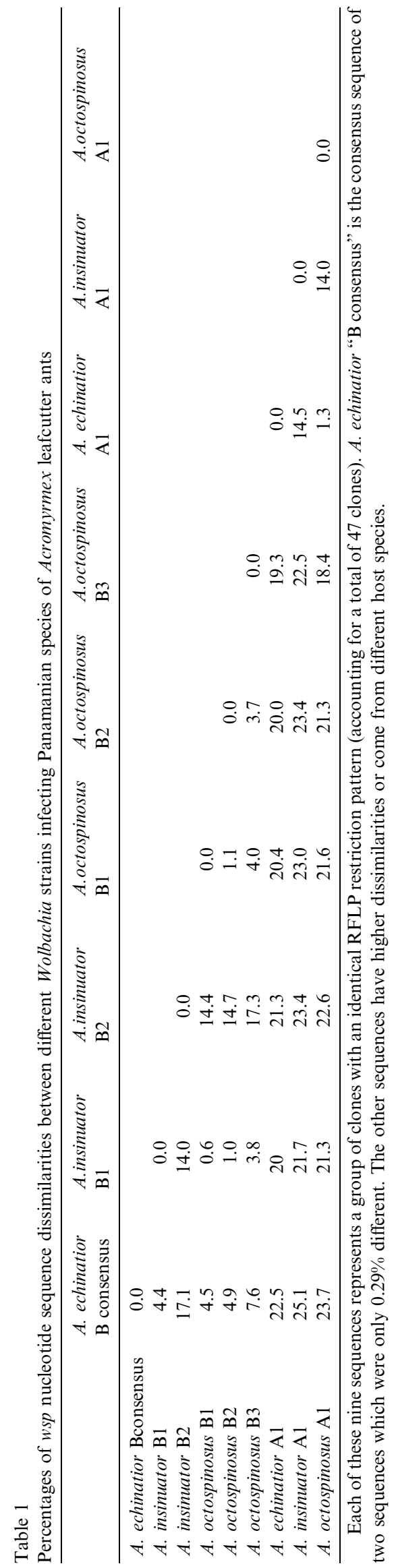

and Solignac, 1995; Wenseleers et al., 1998; Werren et al., 1995a, b; Werren and Windsor, 2000) or more rarely, triple infections (Kondo et al., 2002; Vavre et al., 1999). A stable triple Wolbachia infection has also been created artificially by microinjection of an additional strain in double infected Drosophila (Rousset et al., 1999).

The $w s p$ sequencing as performed here was able to amplify a greater diversity of strains than could be obtained from 16S rDNA amplification (Van Borm et al., 2001). The $w s p$ gene was particularly effective in detecting the four different B strains, all belonging to the InvB subgroup, in A. octospinosus and A. echinatior, whereas none were found in these species using B group-specific 16S rDNA primers (Van Borm et al., 2001). It is likely that this is due to the greater stringency of the strain specific 16S rDNA primers used by Van Borm et al. (2001) relative to the general wsp primers adopted in this study. Indeed, similar differences in specificity between different primer sets have also been observed in other studies. For example, Zhou et al. (1998) could not amplify B group Wolbachia from $N$. vitripennis strains using wsp primers, even though these were known to be present based on $f t s Z$ and $16 \mathrm{~S}$ rDNA sequencing studies (Breeuwer et al., 1992; Werren et al., 1995b).

The multitude of infections demonstrated from wsp sequencing suggests that the Acromyrmex ants must have acquired their Wolbachia during a series of horizontal transmission events. Horizontal transmission is also evident from the fact that strains belonging to four different Wolbachia subgroups (InvA, InsA, Naw, and InvB) are present in three closely related Acromyrmex host species and, reversely, that closely related strains are present in distantly related host species (the InvA and InvB Wolbachia of Acromyrmex are both closely related to Wolbachia found in Solenopsis fire ants, Fig. 1). Earlier, horizontal transmission has been inferred from a lack of congruence between host and Wolbachia phylogenies (Schilthuizen and Stouthamer, 1997) and from documented transmissions in vivo (Huigens et al., 2000). Horizontal transmission may even occur across different insect orders (Van Meer and Stouthamer, 1999; Werren et al., 1995b). Parasitoids have been suggested to be a major route of horizontal transmission, and both transmission from host to parasitoid (Heath et al., 1999) and the reverse (Van Meer and Stouthamer, 1999) have been documented. As ants are known to host a wide range of parasitoids (SchmidHempel, 1998), it may be that they acquire their $\mathrm{Wol}$ bachia in a similar way.

The similarity between two of the strains found in $A$. octospinosus (InvA and InvB) and those found earlier in Solenopsis fire ants (Shoemaker et al., 2000) is intriguing. Possible explanations are that: (1) these Wolbachia strains are specialised to ant hosts (or more specifically, 
ants hosts of the subfamily Myrmicinae to which both Acromyrmex and Solenopsis belong), (2) they were transmitted directly between them by horizontal transmission or (3) they were transmitted from another insect, e.g., a parasitoid, carrying a common Wolbachia ancestor. Direct transmission or transmission via a parasitoid is unlikely, however, since Acromyrmex and Solenopsis occur together in only a very small part of their range in Brazil, whereas introduced fire ants in North America lack Wolbachia infections (possibly due to a bottleneck effect, Shoemaker et al., 2000) and have a distribution that does not overlap with the most northerly distributed Acromyrmex species in Mexico and Arizona (J.T. Longino, pers. comm.). Nevertheless, direct or indirect transmission of Wolbachia between fire ants and leafcutter ants in the southern part of their range cannot strictly be excluded, as a number of diapriid wasps and phorid flies belonging to the same genera are known to infect both Solenopsis spp. and Acromyrmex spp. (Schmid-Hempel, 1998 and references therein). However, adaptation to the physiology of a specific ant host seems more likely, and would be in agreement with the general pattern that other ants tend to be infected by a limited set of closely related $\mathrm{Wol}$ bachia strains (Wenseleers et al., 2001). Host specialisation has also been suggested for parthenogenesis inducing Wolbachia of Trichogramma wasps, where a single wsp clade occurs in nine different host species (Schilthuizen and Stouthamer, 1997), and for feminizing Wolbachia of isopods, where a similar clustering within the Wolbachia phylogeny has been shown (Bouchon et al., 1998).

Sometimes the Acromyrmex species also share closely related strains (the InvB strains in echinatior, insinuator, and octospinosus and the InvA strains in echinatior and octospinosus) and in this case it cannot unequivocally be inferred that the bacteria were inherited from a common ancestor or acquired by horizontal transmission, after speciation had occurred. If Wolbachia was present already before speciation, the estimated divergence time between the Wolbachia strains should accurately reflect the time of host speciation. For the InvB insinuator and echinatior Wolbachia the estimated divergence time based on wsp sequence differences is 1.04 MY (Fig. 1), which agrees reasonably well with the 2 MY divergence time inferred from mitochondrial DNA sequencing (S.R. Sumner et al., manuscript in prep.). Nevertheless, the co-occurrence of several InvB infections in octospinosus suggests that horizontal transmission must also have been important.

The highly complex pattern of Wolbachia infections in leafcutter ants revealed in this study significantly complicates inferences about the potential effects of these symbionts on their host's reproduction. Previously, the A strain Wolbachia of A. octospinosus and A. echinatior (the InvA strains identified here, Fig. 1) has been found to be more abundant in gynes than in males (Van Borm et al., 2001). This observation has been taken as indirect support for an effect on the host's sex ratio via partial male killing (Van Borm et al., 2001). The A strain infection of $A$. insinuator (the InsA strain identified here), on the other hand, has been found to be equally common in males and gynes (Van Borm et al., 2001), which precludes any effects on the host's sex ratio, and suggests that it may have other effects, such as cytoplasmic incompatibility (Van Borm et al., 2001) or enhanced fecundity (Dedeine et al., 2001). A hypothetical prediction about the phenotypes associated with the B strains is more difficult, because multiple B strains occur in each host, and no information exists on the prevalence of each of the strains. Nevertheless, a theoretical analysis by Frank (1998) has predicted that a class of multiply infected individuals can spread in a population of singly infected infividuals only when double infected males are incompatible with single infected females of both strain types. This strengthens the idea that some of the identified strains could be of the incompatibility type.

If future experiments confirm that some of the $A$. Wolbachia do cause incompatibility, then this could have important implications. For example, it could explain how the social parasite $A$. insinuator could have evolved from its host, A. echinatior (Bekkevold and Boomsma, 2000; Schultz et al., 1998) by sympatric speciation. The close phylogenetic affiliation of ant social parasites and their hosts (Schultz et al., 1998) is known as 'Emery's rule' (Baur et al., 1995, 1996; Buschinger, 1986) and while sympatric speciation is often invoked to explain this pattern (Baur et al., 1995, 1996; Buschinger, 1986), it hard to see how it could arise in the absence of a clear mechanism. Incompatibility between multiple Wolbachia types within a population could provide such a mechanism (Hurst and Schilthuizen, 1998; Stouthamer et al., 1999; Werren, 1998). Given that $A$. insinuator carries two additional, phylogenetically isolated,Wolbachia infections relative to its host, $A$. echinatior (Fig. 1) and that Wolbachia-induced speciation has now been well documented in Nasonia (Bordenstein et al., 2001), this scenario deserved further investigation.

\section{Acknowledgments}

We thank the Smithsonian Tropical Research Institute for facilities during field work and the Autoridad Nacional del Ambiente (ANAM) for permission to sample ants in Panama. We thank J. Bot, M. Brown, and S. Sumner for help in the field and B. Hellemans for advice on some of the molecular work. D. Aanen, M. Dijkstra, P. Gertsch, A. Herre, W. Hughes, D. Nash, 
and S. Sumner gave constructive comments on an earlier version of the manuscript. Research in Leuven was supported by Grant No. 981085 from the Institute for the Promotion of Innovation by Science and Technology in Flanders (IWT) to S.V.B. and Grant No. G.0357.99 from the Fund for Scientific Research in Flanders (F.W.O. Vlaanderen) to J.B. and T.W. Fieldwork was supported by a grant of the Danish Natural Science Research Council to J.J.B. The research collaboration between the Universities of Leuven and Copenhagen has been supported by a subcontract under the EU research-training networks "Social Evolution" and "INSECTS."

\section{References}

Arakaki, N., Noda, H., Yamagishi, K., 2000. Wolbachia-induced parthenogenesis in the egg parasitoid Telenomus nawai. Entomol. Exp. Appl. 96, 177-184.

Bandi, C., Anderson, T.J.C., Genchi, C., Blaxter, M.L., 1998. Phylogeny of Wolbachia in filarial nematodes. Proc. R. Soc. Lond. B Biol. Sci. 265, 2407-2413.

Baur, A., Chalwatzis, N., Buschinger, A., Zimmermann, F.K., 1995. Mitochondrial DNA sequences reveal close relationships between social parasitic ants and their host species. Curr. Genet. 28, 242-247.

Baur, A., Sanetra, M., Chalwatzis, N., Buschinger, A., Zimmermann, F.K., 1996. Sequence comparisons of the internal transcribed spacer region of ribosomal genes support close relationships between parasitic ants and their respective host species (Hymenoptera: Formicidae). Insectes Soc. 43, 53-67.

Bekkevold, D., Boomsma, J.J., 2000. Evolutionary transition to a semelparous life history in the socially parasitic ant Acromyrmex insinuator. J. Evol. Biol. 13, 615-623.

Bordenstein, S.R., O'Hara, F.P., Werren, J.H., 2001. Wolbachiainduced incompatibility precedes other hybrid incompatibilities in Nasonia. Nature 409, 707-710.

Bouchon, D., Rigaud, T., Juchault, P., 1998. Evidence for widespread Wolbachia infection in isopod crustaceans: molecular identification and host feminization. Proc. R. Soc. Lond. B Biol. Sci. 265, 10811090.

Braig, H.R., Zhou, W., Dobson, S.L., O’Neill, S.L., 1998. Cloning and characterization of a gene encoding the major surface protein of the bacterial endosymbiont Wolbachia pipientis. J. Bacteriol. 180, 2373-2378

Breeuwer, J.A.J., Stouthamer, R., Burns, D.A., Pelletier, D.A., Weisburg, W.G., Werren, J.H., 1992. Phylogeny of cytoplasmic incompatibility microorganisms in the parasitoid wasp genus Nasonia (Hymenoptera: Pteromalidae) based on 16S ribosomal DNA sequences. Insect Mol. Biol. 1, 25-36.

Buschinger, A., 1986. Evolution of social parasitism in ants. Trends Ecol. Evol. 1, 155-160.

Dedeine, F., Vavre, F., Fleury, F., Loppin, B., Hochberg, M.E., Boulétreau, M., 2001. Removing symbiotic Wolbachia bacteria specifically inhibits oogenesis in a parasitic wasp. Proc. Natl. Acad. Sci. USA 98, 6247-6252.

Felsenstein, J., 1995. PHYLIP: Phylogenetic Inference Package, ver. 3.57c. Department of Genetics, University of Washington, Seattle.

Frank, S.A., 1998. Dynamics of cytoplasmic incompatibility with multiple Wolbachia infections. J. Theor. Biol. 192, 213-218.

Heath, B.D., Butcher, R.D.J., Witfield, W.G.F., Hubbard, S.F., 1999. Horizontal transfer of Wolbachia between phylogenetically distant insect species by a naturally occurring mechanism. Curr. Biol. 9, 313-316.
Hoffmann, A.A., Turelli, M., 1997. Cytoplasmic incompatibility in insects. In: O'Neill, S.L., Hoffmann, A.A., Werren, J.H. (Eds.), Influential Passengers. Inherited Microorganisms and Arthropod Reproduction. Oxford University Press, Oxford, pp. 42-80.

Huigens, M.E., Luck, R.F., Klaassen, R.H., Maas, M.F., Timmermans, M.J., Stouthamer, R., 2000. Infectious parthenogenesis. Nature 405, 178-179.

Hurst, G.D.D., Schilthuizen, M., 1998. Selfish genetic elements and speciation. Heredity $80,2-8$.

Jeyaprakash, A., Hoy, M.A., 2000. Long PCR improves Wolbachia DNA amplification: $w s p$ sequences found in $76 \%$ of 63 arthropod species. Insect Mol. Biol. 9, 393-405.

Kondo, N., Ijichi, N., Shimada, M., Fukatsu, T., 2002. Prevailing triple infection with Wolbachia in Callosobruchus chinensis (Coleoptera: Bruchidae). Mol. Ecol. 11, 167-180.

Kumar, S., Tumara, K., Jakobsen, I.B., Nei, M., 2001. MEGA2: molecular evolutionary genetics analysis software. Bioinformatics (in press).

Malloch, G., Fenton, B., Butcher, R.D., 2000. Molecular evidence for multiple infections of a new subgroup of Wolbachia in the European raspberry beetle Byturus tomentosus. Mol. Ecol. 9, 77-90.

Masui, S., Sasaki, T., Ishikawa, H., 1997. GroE-homologous operon of Wolbachia, an intracellular symbiont of arthropods: a new approach for their phylogeny. Zool. Sci. 14, 701-706.

O’Neill, S.L., Hoffmann, A.A., Werren, J.H., 1997. Influential Passengers: Inherited Microorganisms and Arthropod Reproduction. Oxford University Press, Oxford.

Pintureau, B., Chaudier, S., Lassablière, F., Charles, H., Grenier, S., 2000. Addition of $w s p$ sequences to the Wolbachia phylogenetic tree and stability of the classification. J. Mol. Evol. 51, 374-377.

Rigaud, T., 1997. Inherited microorganisms and sex determination of arthropod hosts. In: O’Neill, S.L., Hoffmann, A.A., Werren, J.H. (Eds.), Influential Passengers. Inherited Microorganisms and Arthropod Reproduction. Oxford University Press, Oxford, pp. 81101.

Rokas, A., 2000. Wolbachia as a speciation agent. Trends Ecol. Evol. $15,44-45$.

Rousset, F., Solignac, M., 1995. Evolution of single and double Wolbachia symbioses during speciation in the Drosophila simulans complex. Proc. Natl. Acad. Sci. USA 92, 6389-6393.

Rousset, F., Braig, H.R., O’Neill, S.L., 1999. A stable triple Wolbachia infection in Drosophila with nearly additive incompatibility effects. Heredity 82, 620-627.

Schilthuizen, M., Stouthamer, R., 1997. Horizontal transmission of parthenogenesis-inducing microbes in Trichogramma wasps. Proc. R. Soc. Lond. B Biol. Sci. 264, 361-366.

Schmid-Hempel, P., 1998. Parasites in Social Insects. Princeton University Press, Princeton.

Schultz, T.R., Bekkevold, D., Boomsma, J.J., 1998. Acromyrmex insinuator new species: an incipient social parasite of fungusgrowing ants. Insectes Soc. 45, 457-471.

Shoemaker, D.D., Ross, K.G., Keller, L., Vargo, E.L., Werren, J.H., 2000. Wolbachia infections in native and introduced populations of fire ants (Solenopsis spp.). Insect Mol. Biol. 9, 661-673.

Stevens, L., Giordano, R., Fialho, R.F., 2001. Male-killing, nematode infections, bacteriophage infection, and virulence of cytoplasmic bacteria in the genus Wolbachia. Annu. Rev. Ecol. Syst. 32, 519545.

Stouthamer, R., 1997. Wolbachia-induced parthenogenesis. In: O’Neill, S.L., Hoffmann, A.A., Werren, J.H. (Eds.), Influential Passengers. Inherited Microorganisms and Arthropod Reproduction. Oxford University Press, Oxford, pp. 102-124.

Stouthamer, R., Breeuwer, J.A., Hurst, G.D., 1999. Wolbachia pipientis: microbial manipulator of arthropod reproduction. Annu. Rev. Microbiol. 53, 71-102.

Thompson, J.D., Higgins, D.G., Gibson, T.J., 1994. CLUSTAL W: improving the sensitivity of progressive multiple sequence align- 
ment through sequence weighting, positions-specific gap penalties and weight matrix choice. Nucleic Acids Res. 22, 6008-6013.

Van Borm, S., Wenseleers, T., Billen, J., Boomsma, J.J., 2001. Wolbachia in leafcutter ants: a widespread symbiont that may induce male killing or incompatible matings. J. Evol. Biol. 14, 805814.

Van Meer, M.M., Stouthamer, R., 1999. Cross-order transfer of Wolbachia from Muscidifurax uniraptor (Hymenoptera: Pteromalidae) to Drosophila simulans (Diptera: Drosophilidae). Heredity 82, $163-169$.

Vandekerckhove, T.T., Watteyne, S., Willems, A., Swings, J.G., Mertens, J., Gillis, M., 1999. Phylogenetic analysis of the 16S rDNA of the cytoplasmic bacterium Wolbachia from the novel host Folsomia candida (Hexapoda, Collembola) and its implications for wolbachial taxonomy. FEMS Microbiol. Lett. 180, 279-286.

Vavre, F., Fleury, F., Lepetit, D., Fouillet, P., Bouletreau, M., 1999. Phylogenetic evidence for horizontal transmission of Wolbachia in host-parasitoid associations. Mol. Biol. Evol. 16, 1711-1723.

Wenseleers, T., Billen, J., 2000. No evidence for Wolbachia-induced parthenogenesis in the social Hymenoptera. J. Evol. Biol. 13, 277280.

Wenseleers, T., Ito, F., Van Borm, S., Huybrechts, R., Volckaert, F., Billen, J., 1998. Widespread occurrence of the micro-organism Wolbachia in ants. Proc. R. Soc. Lond. B Biol. Sci. 265, 1447-1452.

Wenseleers, T., Sundström, L., Billen, J., 2002. Deleterious Wolbachia in the ant Formica truncorum. Proc. R. Soc. Lond. B Biol. Sci. 269, 623-629.
Wenseleers, T., Van Borm, S., Billen, J., Buschinger, A., Boomsma, J.J., 2001. Wolbachia in European ants: incidence and molecular phylogeny. In: Wenseleers, T. (Ed.), Conflict from cell to colony, Ph.D. Thesis. K.U. Leuven, pp. 115-137.

Werren, J.H., 1997. Biology of Wolbachia. Annu. Rev. Entomol. 42, 587-609.

Werren, J.H., 1998. Wolbachia and speciation. In: Howard, D., Berlocher, S. (Eds.), Endless Forms: Species and Speciation. Oxford University Press, New York, pp. 245-260.

Werren, J.H., Windsor, D., Guo, L.R., 1995a. Distribution of Wolbachia among neotropical arthropods. Proc. R. Soc. Lond. B Biol. Sci. 262, 197-204.

Werren, J.H., Zhang, W., Guo, L.R., 1995b. Evolution and phylogeny of Wolbachia: reproductive parasites of arthropods. Proc. R. Soc. Lond. B Biol. Sci. 261, 55-63.

Werren, J.H., Windsor, D.M., 2000. Wolbachia infection frequencies in insects: Evidence of a global equilibrium? Proc. R. Soc. Lond. B Biol. Sci. 267, 1277-1285.

Werren, J.H., Zhang, W., Guo, L.R., 1995. Evolution and phylogeny of Wolbachia-reproductive parasites of arthropods. Proc. R. Soc. Lond. B Biol. Sci. 261, 55-63.

West, S.A., Cook, J.M., Werren, J.H., Godfray, H.C.J., 1998. Wolbachia in two insect host-parasitoid communities. Mol. Ecol. 7, 1457-1465.

Zhou, W., Rousset, F., O'Neill, S., 1998. Phylogeny and PCR-based classification of Wolbachia strains using wsp gene sequences. Proc. R. Soc. Lond. B Biol. Sci. 265, 509-515. 